\title{
COMMENTAIRES SUR LE LIVRE DE SYLVIA OSTROWETSKY
}

\section{Christiane Girard*}

Vivre n'est pas un art mineur.

Christiane Girard

«Sylvia, j'ai enfin pu lire le livre ${ }^{1}$ que tu as écris sur ton pére.. »

Il y a des amis avec qui on vit toute la vie, on est mélées indiscutablement, Il y a les amies qui traversent votre vie, que l'on oublie pas et les auteurs et /ou personnages que de tant avoir aimé une presque intimité s'est installée entre eux et soi.

Sylvia, Je l'ai connue lors de son premier voyage à Brasilia. Un ami comum nous avait demandé de la recevoir. Elle ne connaissait personne dans cette ville et était terrorisée par un précédent accident d'avion. Tout de suite, elle a revélé une immense vitalité et sensibilité. Sur le chemin de la maison, elle nous demandait presque en criant, ce qui était surprenant puisque nous ne nous connaissions pas : « arretezvous! » et elle pleurait d'émotion devant le congrés. « J'ai tellement parlé à mes étudiants de cet endroit... Je revais de le voir! » Ses pleurs, son émotion, chaque jour nous rapprochait.A la fin de son séjour, elle me regarde émue et me dit, je voudrais te donner quelque chose vraiment... Elle retire sa veste qu'elle me donne comme on donne le bijou que l'on a au doigt. A sa seconde visite, elle vient avec son amie algérienne. J'avais traduis une partie de ses cours et traduisis la conférence de son amie. A un certain moment, traduisant l'horreur de la situation en Algérie et les tortures, j'ai du interrompre notre travail. L'émotion était trop forte... On s'est croisée marquée par

\footnotetext{
Doutora, professora do Departamento de Sociologia da Universidade de Brasília (UnB).
}

Artigo recebido em 18 set. 2004; aprovado em 22 out. 2004. 
l'émotion. Le théme qu'elle travaillait: les representations, c'est aussi ce qui m'interessait et même absente, sa présence en moi est vive.

Silvia me parlait de ce livre et il a toujours provoqué en moi un echo...

Moishe et Czarna sa femme sont juifs polonais à Paris. Paris et les étrangers, les étrangers et Paris. Je balance entre les souvenirs juifs polonais de mon quartier pendant l'enfance, la réalité de la guerre d'Algérie et des années suivantes, la «france profonde » et mon quotidien de petite parisienne, radicalement parisienne au milieu de tous ces étrangers qui font partie de mon univers de référence affective, esthétique et morale... Pour tout cela, ce livre me parle, mais aussi pour les thémes qui l'habitent. Ce livre, «C'est de l'or» disait Moïshe, il avait raison. Il nous légue son regard; moqueur, narquois, sensible, sur le monde et sa vie mais aussi celui épouvanté, hanté par les tortionnaires du passé qui nous rattrappent... presque toujours malgré les fleurs, la douceur de l'air et le printemps qui revient.

À mon avis, il s'agit du théme principal, comment vivre quand on a vécu l'epouvante, un peu comme dans les livres de Isaac Singer par exemple, trois amours à New York.

Sauf que dans les livres de Singer, on ne peut pas vivre sans que la Shoa soit constamment présente. C'est un devoir envers les morts, vivre pour faire vivre les morts. Que personne n'oublie! C'est parfaitement juste et parfaitement douloureux. Le sens de vivre: donner du sens à la mort.

C'est ce qu'a fait Sylvia-Zissl, c'est l'hommage de l'enfant à son pére, de toute éternité, reconnaitre la place du pére en temps qu' ordre possible dans la tension du sens qui nous échappe dans la trajectoire humaine. Sylvia dans ce livre reaffirme que son pére lui légue l'essentiel. Vivre n'est pas survivre. C'est aussi pour cette raison qu'il nous parle au coeur. Le pére nomme le fils, lui désigne un lieu d'existence, le fils reconnait le pére et le reconnaitre est un fil pour comprendre le monde qui par ailleurs nous échappe. Peut être 
pourrait-on penser que dans les rapports pére-fille ou mére-fils, le surcroit d'amour et de désir illumine la representation du monde et crée de multiples lectures. La lecture que nous propose Sylvia de Moische tient des deux points d'observation. Il y aurait sans doute d'autres points de lecture mais nous partons de ceux-ci, Sylvia comme fille aimante et admirative et Sylvia, intellectuelle. L'une construit l'autre.

Vivre n'est pas un art mineur... Moishe avait du talent pour vivre. Est-ce que le talent n'existe que pour l'art? Les doués ont un don mais pour que le génie apparaisse, il faut de la discipline, du travail et sans une injonction intérieure, nous n'aurions que du bon travail. On peut dire que pour les intellectuels, il s'agit de la différence entre raisonner et penser. Il est possible de raisonner juste mais penser... exige entre autres obligations, savoir preserver l'emotion, l'intuition et la rigueur... La nécessité de l'art ne laisse la place à aucun autre choix. Il faut de la passion, au sens propre du «pathos » et être au plus prês de son emotion. Pouvoir la transfigurer mais avant la reconnaitre. Moishe n'a pas seulement vécu, il a choisit de vivre.

Vivre est un risque permanent et comme il faut du courage pour assumer ce choix. «Vivre debout » disaient les révolutionnaires même si l'on en finit pas d'avoir peur. Le talent de vivre, c'est un savoir que l'on va chercher en soi, dont la clef ne nous a pas été donnée. Un savoir qui se confronte sans cesse aux autres, à la vie. Moishe avait compris l'essentiel. Il faisait de la vie, de sa vie, son oeuvre d'art L'acharnement et la souplesse mélés.

D'aucuns ont dit que nous avons plus peur d'être heureux que malheureux, Nous en savons la pertinence. L'empirique abonde de données. Moishe nous enseigne ce choix opposé. Vaincre la peur d'être heureux. Il faut trouver des stratégies, des formes quand on retient ce fil si fragile, ce choix si menacé par l'histoire de chacun. Créer c'est donner une forme. Faire ce choix c'est inventer des stratégies qui n'ont jamais existées auparavant, à la mesure de chacun. 
Moishe crée un jardin. Il assemble son histoire, un puzzle, une sculpture, que voit-on de sa vie? Comme dirait un grand ami, « une vie qui tient fort avec des bouts de ficelles ». Que voit-on de sa vie? tout est important comme tout ce qu'il rassemble dans son jardin de banlieue. Un bric à brac de choses apparemment inutiles mais qu'il organise comme un musée vivant.

Qu'est-ce qu'un pére peut enseigner? qu' est-ce qu 'une longue vie pleine d'expériences si elle ne vous donne pas la supériorité du savoir ? C'est ce que Sylvia atteste, Moishe lui a enseigné quelques choses importantes. Des bouts de ficelles qui font tenir l'edifice. Le courage de vouloir être heureux. Sylvia nous le dit quand elle dit: «Ce texte voudrait surtout être heureux».

\section{Le savoir du citoyen et l'ironie tendre de Moishe}

Quand on ne connait pas les régles, les codes, les mots du savoir local sur lesquels bute tout étranger, il faut mobiliser six sens au moins à la fois. Surtout lorsqu'on décide de vivre et non pas de survivre. Que faut-il donc faire? inventer! Moishe invente. En inventant, il construit des ponts sur l'abime, Il saute, le voila sauvé. Comment apprendre le code de la route quand on ne sait pas lire dans sa propre langue? En faisant des pirouettes si énormes que l'on ne peut douter qu'elles sont les pirouettes de celui qui sait puisqu'il use de tant d'assurance! Quand l'examinateur lui demande: que fautil faire pour dépasser une autre voiture, il répond avec un air complice envers l'examinateur: «Il est plus prudent de ne pas dépasser! ». Que faire lorsque l'on est petit et qu'on épouse une femme si belle et décidée? On s'invente d'autres belles femmes qui vous auraient aimé dans la jeunesse, on n'abandonne pas le terrain, on garde sa dignité sans jamais laissé pourtant de trouver sa CZARNA la plus belle de toute, sa complice mais sans abandonner sa force: être debout même petit.

Il faut resister par le récit et de nouvelles pratiques. Il ne voulait pas se montrer sous un mauvais jour. Lutter pour preserver sa dignité, 
c'est vraiment lutter pour son amour. Un amour ou l'on occupe un lieu moindre ne peut résister avec beauté. CZARNA était une femme forte, régie par la précision. Elle se disputait avec lui sur la vérité de leur propre histoire. Moishe opte pour une mémoire qui l'arrange. Ni l'un ni l'autre n'ont tord, ils choissent des repéres différents. Les deux sont entétés, c'est une modalité de relation ou se vit les différences. On explose, la méchanceté est dite, les enfants entrent dans la danse. Le prix est différent pour chacun, entre parent, c'est un rapport d'egalité, avec les enfants, de hierarchie même si ceux-ci ne sont pas dupes de ce qui est en jeu et l'acceptent...

Les enfants apprennent dans le pays d'adoption des parents, ils peuvent parler, écrire avec la désinvolture que les parents n' auront jamais. Mais Moishe n'abandonnera pas sa place: «Ele é o dono da história ». Il est celui qui sait même contre ce que disent les livres, contre ce que disent les professeurs des enfants. Ce qu'il sait, il l'a vécu, construit.Il s'est approprié le savoir du monde. Et quand l'histoire ne lui convient pas, il la change.

C'est pourquoi, il pouvait raconter inlassablement sa vie. Ce n'était jamais exactement la même. Est-ce cette attitude qui lui a permis de vivre le neuf et d'échapper à son passé, sans jamais le renier? en l'éclairant d'un nouveau regard? Pour continuer de vivre, ne faut-il pas accepter le passé sans le tranformer en imuable. Pour vivre aujourd'hui nous changeons et notre regard sur la passé aussi. Peut être est-ce le seul moyen pour que le passé n'alourdisse le présent jusqu'à le tuer. Dans les histoires ténébreuses de son enfance en Pologne ou du nazisme et des fuites et de l'exode, il se souvenait particuliérement et privilégiait aussi son agilité, ses ressources pour échapper au mal absolu. Il cherchait la lumiére dans l'obscurité et la retrouve en lui ou dans la delicatesse d'un bourgeon.

Dans les romans d'Isaac Singer, les juifs n'ont pas le droit d'oublier. Nous n'avons pas le droit d'oublier. C'est juste. Mais peuton ne pas oublier et vivre?

Moishe nous répond, oui. Il rassemble dans son jardin, l'inatendu, l'insolite, des objets récupérés au jour le jour de son metier 
de chiffonier, des objets absoluments communs, du quotidien. Plante des arbres. Il dit, raconte, construit a travers ce nouveau monde. Il invente un langage. Il ne domine pas la langue française. C'est certainement douloureux aucun étranger n'en sort indemne mais c'est aussi une chance, dominer n'est pas une bonne façon d'aimer. Dans les interstices de l'incertitude, il nous enseigne que, l' histoire se dépasse sans sauter par dessus son ombre.

Moishe e Czarna, deux être qui resistent sans posséder les codes et sans se renier, ils vivent. En faut-il de l'entétement et de l'arrogange. Vouloir avoir raison contre toute évidence, contre ses enfants qui eux étudient et deviennent savants.Mais peut-on être pére et mére sans avoir le dernier mot ? même si les enfants ne se trompent plus? oui Zissl lui accorde, le dernier mot, la place du pére. Il a conquis ce lieu de haute lutte, par son énergie inlassable, sa force et la souplesse de savoir s'adapter sans se renier.

De ce pére aimé et admiré, Zissl et Sylvia nous démontre l'intelligence. Pratiquement illetré, il s'était crée des repéres linguistiques d'une extrême complexité.

Je regarde Sylvia au dela de sa mort qui nous sépare avec tendresse et un immense respect pour son pére et pour elle, je lui dirais si elle était là. Oui, il était passionnant ton pére... Mais c'est un mot qui la couvre aussi dans sa pratique de recherche, d'avoir été une intellectuelle qui a traqué la compréhension de l'ordre du monde en parlant sa propre langue, infatigablement, courageusement et parfois solitairement.

Moishe, malade, ayant perdu celle qu'il aimait, est hospitalisé. Dans un délire, il tombe par la fenêtre. S'est-il suicidé, poursuivi dans son délire par les tortionnaires nazistes? Il me semble que Zissl l'a pensé, certains mots sont rapportés qui le laisse imaginer. Cela signifirait-il que l'on n'échappe pas à son histoire? Les démons hantent nos têtes et nous sommes ou nous ne pensons pas comme disait Lacan (Sylvia a été analysée par Lacan). Sans doute est ce vrai et 
c'est trés douloureux. Mais éveillé, Moishe nous montre que l'on peut vivre debout et que l'on peut en inventant sa langue, sa forme d'être, continuer de crée son histoire.

Moishe me rappelle le peintre Bispo qui assemblaient des morceaux de tissus peints en faisait une cape dont il se couvrait, une cape singuliére et glorieuse ou douleur et bonheur sont étroitement mélés mais n'oublions pas pour cela la lumiére éblouissante de la leçon que Sylvia nous offre. Moishe l'a dit: ce livre c'est de l'or! C'etait quelqu'un Moishe.

\section{Note}

1 Ostrowetsky, Sylvia. Quelqu'un ou le livre de Moïshe. Paris: Kimé, 1995. 
